materials systems to be studied. Currently, the WDM is used as a research tool to determine relationships between the geological structure of the subsurface and fluid-flow phenomena. For example, it produces the correct dynamics of the displacement of one fluid by another, such as oil by brine in an oil reservoir, and groundwater by heavier-than-water contaminants in an aquifer. WDM has also been used to predict the thermal properties of new composite materials proposed for use in nuclear reactors. Theoretical mathematical research such as Van Siclen's work aids in the development of accurate and reliable predictive models.

\section{Switching and Memory Effects due to CDW Deformation Observed in $\mathrm{K}_{0.3} \mathrm{MoO}_{3}$}

Sliding charge-density-wave (CDW) materials exhibit unique memory effects including pulse sign memory, pulse duration memory, and delayed conduction. CDW materials are composed of an elastic periodic medium under the influence of randomly distributed pinning sites. Under an external driving force, $\mathrm{CDW}$ materials show a dynamic phase transition from a creep phase to a slide phase. A strong nonlinear conduction, called switching, corresponds to this transition. As reported in the April 29 issue of Applied Physics Letters, N. Ogawa and K. Miyano of the University of Tokyo have demonstrated electro-optical switch and memory effects in $\mathrm{K}_{0.3} \mathrm{MoO}_{3}$, a CDW material. The researchers established this CDW material as an electrically writable/readable and optically erasable memory device by incorporating only two electrical leads.

The researchers demonstrated optical switching in $\mathrm{K}_{0.3} \mathrm{MoO}_{3}$ by applying an increasing voltage and light illumination to a sample at $12 \mathrm{~K}$. Below the sliding voltage, $V_{\mathrm{S}}$, the current in the sample was caused by the creeping motion of the CDW. At $V_{\mathrm{s}}$, the current in the sample increased by about 3 orders of magnitude. According to the researchers, this observed switching at $V_{\mathrm{s}}$ corresponds to the creep-to-slide dynamic phase transition. The creep current and $V_{\mathrm{s}}$ increase with increasing light illumination, an indication of optical excitation affecting the dynamic phase transition of the CDW.

The researchers also demonstrate conduction delay of the CDW transport. Application of a voltage slightly larger than $V_{\mathrm{s}}$ and a rectangular voltage pulse causes, after a time delay, sliding motion of the CDW. By illuminating the sample before the voltage pulse, there is an increase in the time delay. The delay time depends on the amplitude of the voltage pulse and the illumination intensity. After the initial sliding effect, sliding occurs more quickly when the next voltage step is applied. This is the memory effect.

According to the researchers, these results indicate that the CDW dynamic phase transition from creep phase to slide phase can be set by an external electric field and removed by photoexcitation and that this property can be exploited to realize an electrically readable switch or memory device.

\section{JENNIFER BURRIS}

\section{Low Humidity Content in Soil Detected Using an Indium-Doped Tin Oxide (ITO) Sensor}

Agriculture is not immune to the current global trend of reducing costs to increase profitability. Automatic irrigation systems are currently available to help reach this goal. Some of these systems operate within a scheduled time frame, and others work by controlling the amount of water present in the soil, allowing watering only when it is needed and thus compensating for unexpected changes in weather. The latter can be achieved by using sensors embedded in the soil.

Sensors used to monitor the water content in the soil measure either dielectric permittivity or electrical resistance. Sensors that measure dielectric permittivity are expensive though more reliable, and are typically used for higher water content measurements. Sensors that measure electrical resistance typically measure atmospheric moisture. These are porous materials in which surface adsorption of water molecules in the internal cavities changes the conductivity; therefore, the harshness of the environment determines the capability for monitoring humidity in the soil after long periods of time.

A group of scientists from the Universitat Jaume I in Spain have attempted to develop an inexpensive sensor to provide accurate measurements at low humidity levels in the soil. Their first steps toward developing such a device from indiumdoped tin oxide (ITO) are described in the April 15 issue of Applied Physics Letters. ITO is a wide-bandgap semiconductor with high conductivity. The cost and availability, and chemical stability in water, basic, and acidic solutions, make ITO an excellent choice for this application.

Square pieces of glass $(1.5 \mathrm{~cm} \times 1.5 \mathrm{~cm})$ coated with an ITO film served as electrodes, with a groove $500 \mu \mathrm{m}$ wide dividing the film in half. Silver epoxy fixed the electrical contacts on each half, and a commercial thermoplastic was thermally sealed on top of each electrode. As a first test, this sensor was used to measure the humidity in $2 \mathrm{~kg}$ of soil placed inside a container. The scientists placed the sensor in contact with the soil itself, and then applied a voltage of $100 \mathrm{mV}$ with sinu- soidal amplitude. The ac impedance response obtained had considerable reproducibility at different conditions of dryness and humidity.

At low frequencies, the results show the effect of electrode polarization. At high frequencies, the bulk response results from the combination of a resistance $R_{p}$ and a capacitance $C_{p}$, in parallel. These two values, resolved by the appropriate software, varied with the water content in the soil. The resistance $R_{p}$ decreased about 2 orders of magnitude with an increase in the water content from $\sim 3 \mathrm{wt} \%$ to $10 \mathrm{wt} \%$; the capacitance Cp increased from $1.2 \times 10^{-10} \mathrm{~F}$ to $1.8 \times 10^{-10} \mathrm{~F}$ from driest to water-saturated soil. Since the capacitance is negligible as compared with the values for surface polarization at the Helmholtz layer, $\sim 5 \mu \mathrm{F} / \mathrm{cm}^{2}$, these observations cannot be attributed to a contact effect, said the researchers.

F. Fabregat-Santiago and co-workers said the behavior of the ITO sensor in terms of the two adsorption mechanisms represents chemical adsorption, where the electrostatic field of surface ions attracts water molecules to form the first layer; and physical adsorption, where water molecules dissociate and release protons to form additional layers. In these two cases and at low-humidity levels, the released protons determine the nature of the electrical conductivity. At high humidity levels, water condenses and then electrolytic conduction also contributes to the electrical conductivity. For this reason, a plateau in the conductivity appears at humidity levels of $10 \%$ and higher, according to the researchers. This trend is consistent with measurements conducted in different conditions; in some cases, the value of the resistance increased as expected when the dielectric constant of the medium increased, but the trend remained the same.

The ITO sensor can accurately detect low levels of humidity in the soil, and it addresses the main concerns of cost and reliability of measurements at low humidity. However, the researchers said that further testing is needed under operational conditions in the field to determine the applicability of this device for triggering an automatic irrigation system during extended periods of time.

SIARI S. SOSA

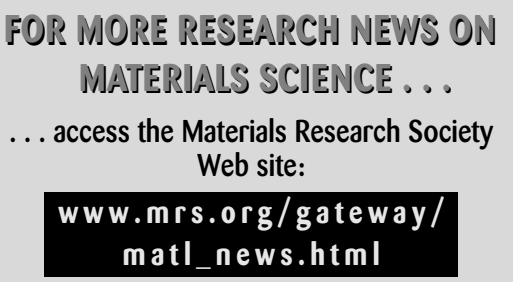

